\title{
Eficacia y seguridad de la vacuna contra el virus de papiloma humano para la prevención del cáncer de cuello uterino: revisión sistemática y metaanálisis Efficacy and safety of human papilloma virus vaccine in cervical cancer prevention: systematic review and meta-analysis
}

\author{
Dra. Lucila Rey-Ares ${ }^{a}$, Dr. Agustín Ciapponi ${ }^{a, b}$ y Prof. Dr. Andrés Pichon-Riviere ${ }^{a, c}$
}

\section{RESUMEN}

Introducción. El cáncer de cuello uterino es el tercer tipo de cáncer en frecuencia en el mundo. El virus de papiloma humano (HPV, por su sigla en inglés) se ha identificado como causa de éste y otros cánceres anogenitales. El objetivo del presente trabajo es evaluar, mediante una revisión sistemática y metaanálisis, la eficacia y seguridad de las vacunas contra HPV para la prevención de neoplasias intraepiteliales (CIN) de grados 2, 3 y adenocarcinoma in situ (CIN2+) y el cáncer de cuello uterino.

Materiales y métodos. Se realizó una búsqueda sistemática en las principales bases de datos durante julio de 2011 sin restricciones temporales o idiomáticas. Se incluyeron ensayos clínicos controlados aleatorizados que evaluaran la eficacia de la vacuna contra el desarrollo de CIN2+ y cáncer de cuello uterino.

Resultados. Se confeccionó un metaanálisis con 4 de las 168 publicaciones halladas. Mediante el análisis por intención de tratar se observó, para mujeres vacunadas, un riesgo relativo (RR) de contraer lesiones CIN2+ asociadas a HPV 16 de 0,45 (IC 95\% 0,38-0,54); a HPV 18 de $0,14(0,08-0,25)$ y por serotipos oncogénicos $31 / 33 / 45 / 52 / 58$ no incluidos en la vacuna de $0,79(0,68-0,93)$. Todos los estudios mostraron perfiles de seguridad aceptables.

Conclusiones. Las vacunas disponibles actualmente mostraron ser eficaces y seguras para la prevención de lesiones $\mathrm{CIN} 2+$; sin embargo, resta probar su eficacia a largo plazo.

Palabras clave: vacunas contra el virus del papiloma humano, cáncer de cuello uterino, mujeres, revisión sistemática y metaanálisis.

http:/ /dx.doi.org/10.5546/aap.2012.483

\section{INTRODUCCIÓN}

El cáncer de cuello uterino constituye un problema de salud para todas las mujeres del mundo y es el tercer tipo de cáncer en frecuencia. Según la base internacional de registro del cáncer GLOBOCAN, ${ }^{1}$ en 2008, 68220 de los 530000 casos diagnosticados en el mundo ocurrieron en Latinoamérica y el Caribe. Más del 85\% de la carga de enfermedad mundial le pertenece a países en desarrollo, donde el cáncer de cuello uterino representa el 13\% de las neoplasias femeninas. Latinoamérica y el Caribe son una región de alto riesgo, con una incidencia (tasa estandarizada por edad) de 23,5/100 000 habitantes y una mortalidad de 10,8/100 000.

La asociación entre el virus del papiloma humano (HPV) y el cáncer de cuello uterino fue sugerida en la década de $1970,{ }^{2}$ luego de lo cual numerosos estudios epidemiológicos y moleculares proveyeron evidencia irrefutable sobre el papel del HPV como agente etiológico del cáncer de cuello uterino y otros cánceres anogenitales que comparten características epidemiológicas con las enfermedades de transmisión sexual. ${ }^{3,4}$ Menos frecuentemente, el HPV se ha asociado con cánceres de la orofaringe y papilomatosis respiratoria.

Más de 35 tipos de HPV infectan el tracto genital, y los más comunes son el 6, 11, 16 y 18. Los dos primeros se consideran de bajo riesgo, porque raramente se los encuentra asociados con cáncer de cuello uterino invasivo, a diferencia de los serotipos 16 y 18, clasificados como de alto riesgo., ${ }^{3,4}$

La infección persistente por HPV puede ocasionar lesiones preneoplásicas; neoplasias intraepiteliales cervicales -CIN (del inglés Cervical Intraepithelial Neoplasia)-, categorizadas de 1 a 3 según criterios histopatológicos; adenocarcinoma in situ (AIS); o cáncer de cuello uterino invasivo. CIN 1 indica la presencia de infección por HPV activa, CIN 2 y 3 son consideradas lesiones premalignas $\mathrm{y}$, por tanto, 
tienen indicación de tratamiento al igual que el AIS y el cáncer de cuello uterino.

Un metaanálisis recientemente publicado ${ }^{5}$ muestra que en Latinoamérica y el Caribe el 82,5\% de las lesiones CIN 2 y 3, y el 89\% de los cánceres de cuello uterino fueron positivos para HPV, con una proporción atribuible a HPV 16/18 similar a la comunicada mundialmente.

Dentro de las medidas implementadas para la prevención del cáncer de cuello uterino, la detección de lesiones cervicales premalignas mediante Papanicolau (PAP test) y colposcopia han demostrado ser intervenciones muy exitosas que lograron disminuir la incidencia y mortalidad en el mundo entero.

Con el objetivo de reducir la carga de enfermedad relacionada con el HPV se han desarrollado vacunas no infecciosas, adyuvadas, compuestas por partículas similares al virus (VLPs, del inglés Virus-Like Particles) de HPV 16, 18, 6 y 11 (tetravalente) y de HPV 18 y 16 (bivalente).

$\mathrm{Su}$ aparición generó un enorme entusiasmo en la comunidad médica y el público en general. En los últimos años se han incorporado en los calendarios oficiales de vacunación de numerosos países, ${ }^{6}$ entre los que se encuentra la Argentina.

Debido a que los casos de cáncer de cuello uterino son infrecuentes, los estudios que evaluaron la eficacia de la vacuna utilizaron como puntos finales subrogantes del cáncer de cuello uterino: la infección persistente por $\mathrm{HPV}$, las lesiones CIN 1, 2, 3 y el AIS, siendo este último uno de los aspectos que más controversias ha generado ${ }^{7,8}$ al momento de interpretar los resultados de eficacia. Tanto la Organización Mundial de la Salud como la FDA (Food and Drug Administration $)^{9-11}$ consideran como puntos finales subrogantes válidos para evaluar la efectividad de las vacunas contra el HPV a las lesiones CIN 2+ (CIN2, CIN3 y AIS).

La vacunación contra el HPV es una intervención importante en salud pública que afecta a un gran porcentaje de la población, su implementación impacta en el presupuesto de los países y genera importantes consecuencias sanitarias a largo plazo, por lo que se vuelve fundamental contar con información sólida para tomar decisiones, sobre todo en países de medianos y bajos ingresos como los de Latinoamérica y el Caribe.

El objetivo del estudio fue evaluar mediante una revisión sistemática y metaanálisis la evidencia disponible acerca de la eficacia y seguridad de la vacuna contra el HPV para la prevención del cáncer de cuello uterino.

\section{MATERIALES Y MÉTODOS}

Se realizó una búsqueda en las bases de datos bibliográficas MEDLINE, Cochrane Central Register of Controlled Trials (CCTR), DARE, NHS EED. Se efectuó además una segunda búsqueda, orientada específicamente a hallar evaluaciones económicas de la vacuna contra el HPV realizadas en países de Latinoamérica y el Caribe en MEDLINE, LILACS, EMBASE y DARE. La búsqueda se complementó en buscadores genéricos de Internet y agencias de evaluación de tecnologías sanitarias con empleo de los términos: Papillomavirus Vaccines, $H P V$ vaccine, Cervarix ${ }^{\circledR}$ y Gardasil $^{\circledR}$.

Se consideraron criterios de inclusión que los estudios fueran ensayos clínicos controlados y aleatorizados, que evaluaran la eficacia de las vacunas bivalentes y tetravalentes. Para la realización de esta revisión sistemática y metaanálisis se tomaron sólo los puntos finales correspondientes a las lesiones CIN2+ o cáncer de cuello uterino, según análisis por intención de tratar.

Dos de los autores (AC, LRA) seleccionaron los estudios en forma independiente y evaluaron su calidad metodológica, las discrepancias se resolvieron por consenso de todo el equipo (AC, APR, LRA). Se empleó el programa Review Manager 5.1 (RevMan). Se utilizó el método de efectos aleatorios para dar cuenta de la posible heterogeneidad clínica o metodológica y, al mismo tiempo, arrojar estimaciones más conservadoras (intervalos de confianza más amplios).

\section{RESULTADOS}

Se identificaron en total 168 artículos, de los cuales se seleccionaron para ser evaluados por texto completo 91 estudios; finalmente, se incluyeron para confeccionar el metaanálisis de eficacia de la vacuna cuatro publicaciones de tres ensayos clínicos controlados y aleatorizados ${ }^{12-15} \mathrm{y}$, para la evaluación de la costo-eficacia de la vacunación en la región, diez evaluaciones económicas (Figura 1).

Los estudios incluidos fueron ensayos clínicos controlados y aleatorizados en fase III, multicéntricos, dos de ellos realizados en América, Asia y Europa, mientras que el restante se realizó sólo en el continente Americano (EE.UU., Canadá y Brasil). Los tres estudios, de alta calidad metodológica, incluyeron en total 190534 mujeres de entre 15 y 25 años sin antecedentes de lesiones genitales o infección por HPV; las asignadas a la rama vacuna recibieron 3 dosis de vacuna bivalente $o$ tetravalente, mientras que las participantes de la rama control recibieron placebo o vacuna contra hepatitis A. La media de seguimiento de todos los 
estudios en conjunto fue de 42,8 meses y se llevaron a cabo entre los años 2003 y 2005 (Tabla 1).

CIN2+ asociados a HPV 16: El riesgo relativo (RR) combinado obtenido del metaanálisis de los 3 ensayos clínicos controlados y aleatorizados que en conjunto sumaron 31955 pacientes en total, fue de 0,45 (IC 95\% 0,38-0,54) en el análisis por intención de tratar, que se corresponde con una reducción del riesgo relativo (RRR) de un $55 \%$ para las mujeres vacunadas. Aunque la heterogeneidad para este metaanálisis es muy importante ( $\left.\mathrm{I}^{2} 91 \%\right)$, el $80 \%$ del peso está dado por el estudio mayor RR (FUTURE I y II) por lo que la estimación central del metaanálisis es la más conservadora, poco influenciada por el resto de los estudios (Figura 2).

CIN2+ asociados a HPV 18: El análisis por intención de tratar sobre un total de 33046 mujeres mostró un beneficio significativo para el grupo vacunado con un riesgo relativo agrupado de 0,14 (IC
95\% 0,08-0,25), que se corresponde con una redución del riesgo relativo (RRR) de sufrir lesiones CIN2+ de un $86 \%$ en las mujeres vacunadas. En este caso la heterogeneidad fue nula $\left(\mathrm{I}^{2} 0 \%\right)$ (Figura 3).

CIN2+ asociados a HPV 31/33/45/52/58: Con respecto a las lesiones ocasionadas por los tipos de HPV oncogénicos no incluidos en la vacuna se determinó mediante el metaanálisis una RRR estadísticamente significativa $(\mathrm{p}=0,004)$ del $21 \%$ (RR 0,79; IC 95\% 0,68-0,93) en el grupo de mujeres vacunadas sobre un total de 34383 participantes. Se observó heterogeneidad significativa con un $\mathrm{I}^{2}$ de $71 \%$. Se estima entonces que existe alrededor de un $20 \%$ de protección cruzada contra los serotipos oncogénicos no incluidos en la vacuna (Figura 4).

Con respecto a la seguridad de las vacunas, todos los estudios mostraron perfiles de seguridad aceptables. En el estudio PATRICIA ${ }^{12}$ no se hallaron diferencias entre el grupo vacunado y

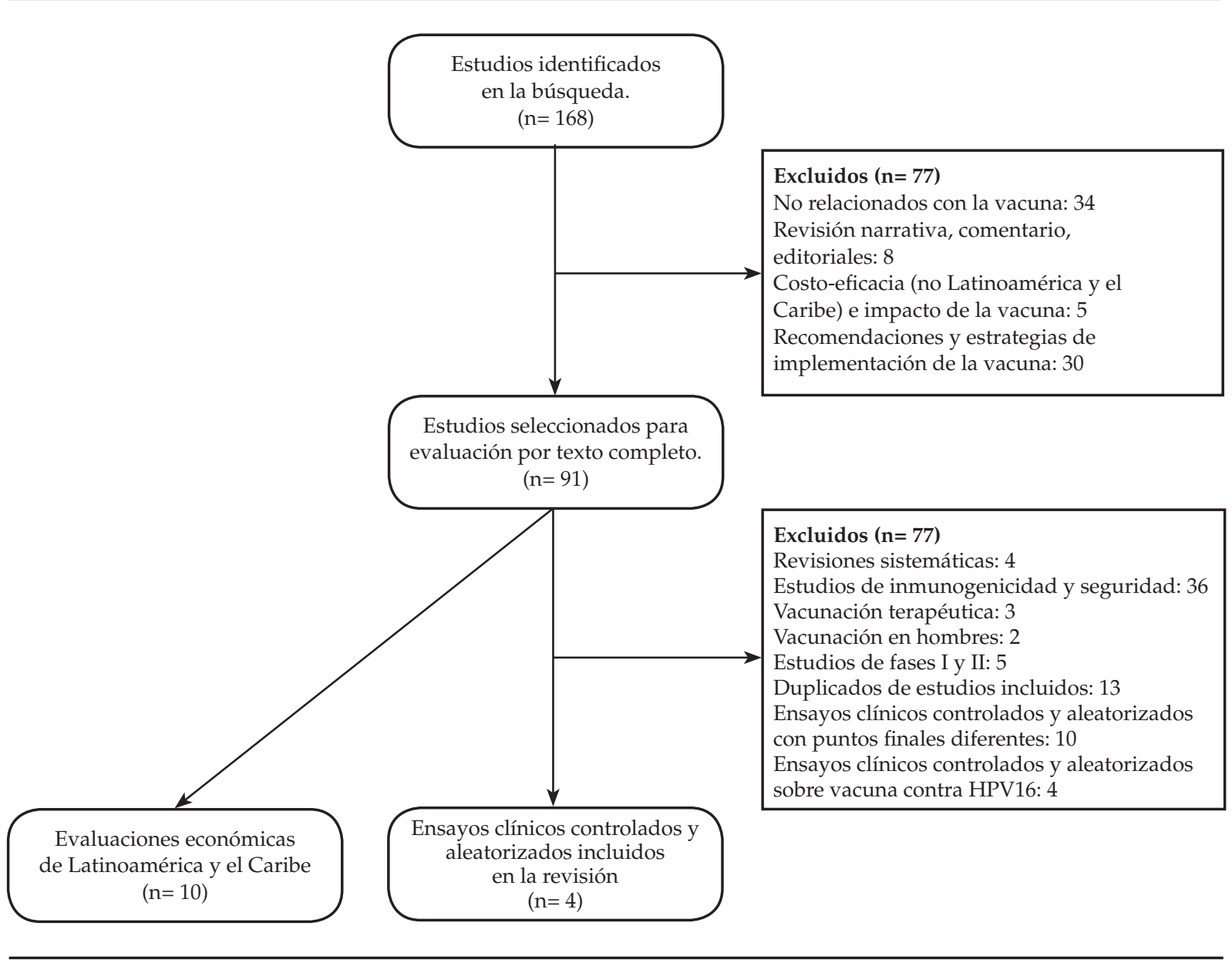


TABLA 1. Características de los estudios incluidos en el metaanálisis

\begin{tabular}{|c|c|c|c|}
\hline & Harper 2006 & FUTURE I \& II & PATRICIA \\
\hline Publicaciones utilizadas & Harper 2006 & Wheeler 2009 y Muñoz 2010 & Paavonen 2009 \\
\hline Fase & III & III & III \\
\hline Cantidad de centros & 28 & $62 / 90$ & 135 \\
\hline Cantidad de países & 3 & $16 / 13$ & 14 \\
\hline Fecha de enrolamiento & $11 / 2003-7 / 2004$ & $1 / 2002-5 / 2003$ & $5 / 2004-6 / 2005$ \\
\hline Financiación & GlaxoSmithKline & Merck & GlaxoSmithKline \\
\hline \multicolumn{4}{|l|}{ Criterios de inclusión } \\
\hline Edad (años) & $16-25$ & $15-26$ & $15-25$ \\
\hline$N^{o}$ de parejas sexuales & $\leq 5$ & $\leq 4$ & $\leq 6$ \\
\hline Criterios de exclusión & $\begin{array}{c}\text { Embarazo o } \\
\text { historia de PAP anormal }\end{array}$ & $\begin{array}{c}\text { Infecciones previas por HPV, } \\
\text { historia de PAP test anormales } \\
\text { o de verrugas genitales }\end{array}$ & $\begin{array}{c}\text { Historia de colposcopía } \\
\text { embarazo, lactancia, } \\
\text { enfermedades crónicas o } \\
\text { autoinmunitarias }\end{array}$ \\
\hline \multicolumn{4}{|l|}{ Intervención y comparador } \\
\hline Componentes de la vacuna & HPV 16, 18 VLPs & HPV $6,11,16,18$ VLPs & HPV 16, 18 VLPs \\
\hline Comparador & Placebo & Placebo & Vacuna contra hepatitis A \\
\hline Esquema de administración & 0,1 y 6 meses & 0,2 y 6 meses & 0,1 y 6 meses \\
\hline \multicolumn{4}{|l|}{ Protocolo de seguimiento } \\
\hline Búsqueda de ADN viral & cada 6 meses & cada 6 meses & cada 6 meses \\
\hline PAP & cada 6 meses & cada $6 / 12$ meses & cada 12 meses \\
\hline Tiempo de seguimiento (meses) & $\begin{array}{c}\text { Estudio inicial } 27 \text { y } \\
\text { estudio de seguimiento } 53\end{array}$ & 36 (media) & 39,4 (media) \\
\hline
\end{tabular}

Puntos finales de resultado

\section{Primarios}

Secundarios
Infección persistente por HPV 16 y/o 18. LSIL, HSIL, CIN 1-3 y cáncer de cuello uterino asociado a HPV 16 y/o 18
Incidencia de verrugas genitales, CIN 1-3, VIN 1-3; AIS, cáncer vulvar, asociadas a HPV 16 y/o 18 vaginal o cervical asociados a HPV 6, 11, 16 o 18

Infección persistente, CIN 1-3 y AIS asociados a HPV 31, 33, 45, 52, 58. Incidencia combinada de CIN 1-3, AIS y cáncer por HPV 6, 11, 16 y/o 18.

Infección persistente por HPV

16, 18 u otros tipos oncogénicos, lesiones CIN asociadas a

HPV 16, 18, otros tipos oncogénicos o sin aislamiento de ADN viral. Reducción la realización de colposcopía y procedimientos escisionales.

\section{Población para análisis de eficacia}

Análisis por protocolo

Todas las participantes que recibieron 3 dosis de vacuna o placebo, con prueba de ADN para HPV negativa el día $1 \mathrm{y}$ seronegativas para HPV 16 y/o 18

Sin violaciones al protocolo.

\begin{abstract}
Análisis por intención de tratar
\end{abstract}

\begin{abstract}
Todas las participantes que recibieron al menos 1 dosis de vacuna o placebo, con prueba de ADN para HPV negativa. Con datos disponibles para evaluar eficacia
\end{abstract}

Todas las participantes que recibieron 3 dosis de vacuna o placebo en el lapso de 1 año, seronegativas, con prueba de ADN negativa para HPV 6,

11, 16 o 18 en el día 1 y que mantuvieron la prueba de ADN negativa durante los primeros

7 meses de seguimiento.

Sin violaciones al protocolo.

Todas las participantes aleatorizadas sin importar los antecedentes de

lesiones anogenitales o de infección por HPV.
Todas las participantes que recibieron 3 dosis de vacuna o placebo, con prueba de ADN para HPV negativa, seronegativas para HPV 16 y/o

18 y con citología normal

o de bajo grado el día 1.

Sin violaciones al protocolo.

Todas las mujeres que recibieron al menos una dosis de vacuna o placebo con citología

normal o de bajo grado al día uno y con datos disponibles para evaluar eficacia.

\section{Calidad metodológica}

\begin{tabular}{lc} 
Aleatorización & Adecuada \\
Enmascaramiento & Adecuado \\
Informe de pérdidas de seguimiento & Sí \\
Eficacia esperada (1-RR) & 0,7 \\
Cálculo de tamaño muestral & Sí \\
\hline
\end{tabular}

$\begin{array}{cc}\text { Adecuada } & \text { Adecuada } \\ \text { Adecuado } & \text { Adecuado } \\ \text { Sí } & \text { Sí } \\ 0,80-0,90 & 0,85 \\ \text { Sí } & \text { Sí }\end{array}$

PAP test: Papanicolaou; LSIL: lesión escamosa intraepitelial de bajo grado; HSIL: lesión escamosa intraepitelial de alto grado; CIN: neoplasia intraepitelial cervical; CCU: cáncer de cuello uterino; AIS: adenocarcinoma in situ, VIN: neoplasia intraepitelial vulvar. 
el control en ninguno de los puntos finales considerados. En los dos estudios restantes ${ }^{14,16,17}$ se observó un mayor informe de episodios adversos no graves en el grupo vacunado, siendo los más frecuentes los efectos adversos locales (enrojecimiento, prurito, dolor e hinchazón del sitio de inyección). Ninguno de los estudios halló diferencias entre los grupos en lo que respecta a los episodios adversos graves y episodios adversos relacionados con el embarazo (abortos, malformaciones congénitas, prematurez, etc.).

\section{DISCUSIÓN}

Los programas de prevención primaria orientados a evitar las enfermedades de transmisión sexual y de pesquisa mediante Papanicolau continúan siendo una medida eficaz para la prevención, detección y tratamiento temprano del cáncer de cuello uterino.

El hecho de contar con una vacuna eficaz y segura capaz de prevenir lesiones CIN2+ asociadas a los serotipos 16 y 18 de HPV es prometedor, aunque aún no existe evidencia suficiente que res-

FIGURA 2. CIN2+ asociados a HPV 16

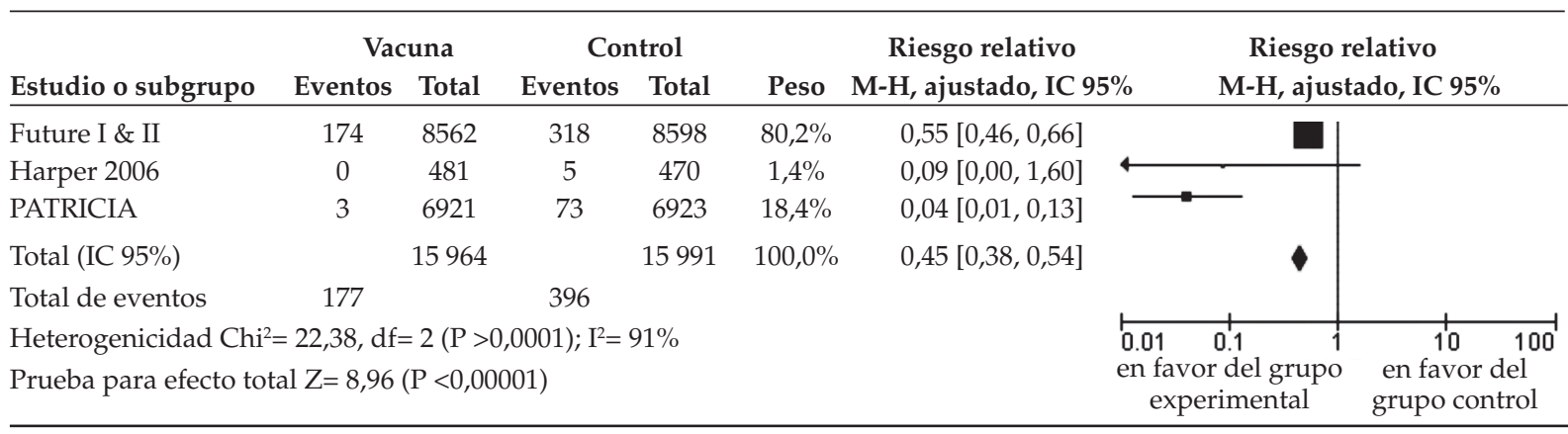

FigURA 3. CIN2+ asociados a HPV 18

\begin{tabular}{|c|c|c|c|c|c|c|c|c|}
\hline \multirow[b]{2}{*}{ Estudio o subgrupo } & \multicolumn{2}{|c|}{ Vacuna } & \multicolumn{2}{|c|}{ Control } & \multirow[b]{2}{*}{ Peso } & \multirow{2}{*}{$\begin{array}{c}\text { Riesgo relativo } \\
\text { M-H, ajustado, IC 95\% }\end{array}$} & \multirow{2}{*}{\multicolumn{2}{|c|}{$\begin{array}{c}\text { Riesgo relativo } \\
\text { M-H, ajustado, IC 95\% }\end{array}$}} \\
\hline & Eventos & Total & Eventos & Total & & & & \\
\hline Future I \& II & 11 & 8562 & 71 & 8598 & $74,7 \%$ & $0,16[0,08,0,29]$ & & \\
\hline Harper 2006 & 0 & 481 & 0 & 470 & & No estimable & & \\
\hline PATRICIA & 2 & 7455 & 24 & 7480 & $25,3 \%$ & $0,08[0,02,0,35]$ & & \\
\hline Total (IC 95\%) & & 16498 & & 16548 & $100,0 \%$ & $0,14[0,08,0,25]$ & & \\
\hline Total de eventos & 13 & & 95 & & & & & \\
\hline \multicolumn{5}{|c|}{ Heterogenicidad $\mathrm{Chi}^{2}=0,60 \mathrm{df}=1(\mathrm{P}=0,44) ; \mathrm{I}^{2}=0 \%$} & & & $0.01 \quad 0.1$ & $10 \quad 100$ \\
\hline \multicolumn{5}{|c|}{ Prueba para efecto total $\mathrm{Z}=6,72(\mathrm{P}<0,00001)$} & & & $\begin{array}{l}\text { en favor del grupo } \\
\text { experimental }\end{array}$ & $\begin{array}{l}\text { en favor del } \\
\text { grupo control }\end{array}$ \\
\hline
\end{tabular}

FigURA 4. CIN2+ asociados a HPV 31/33/45/52/58

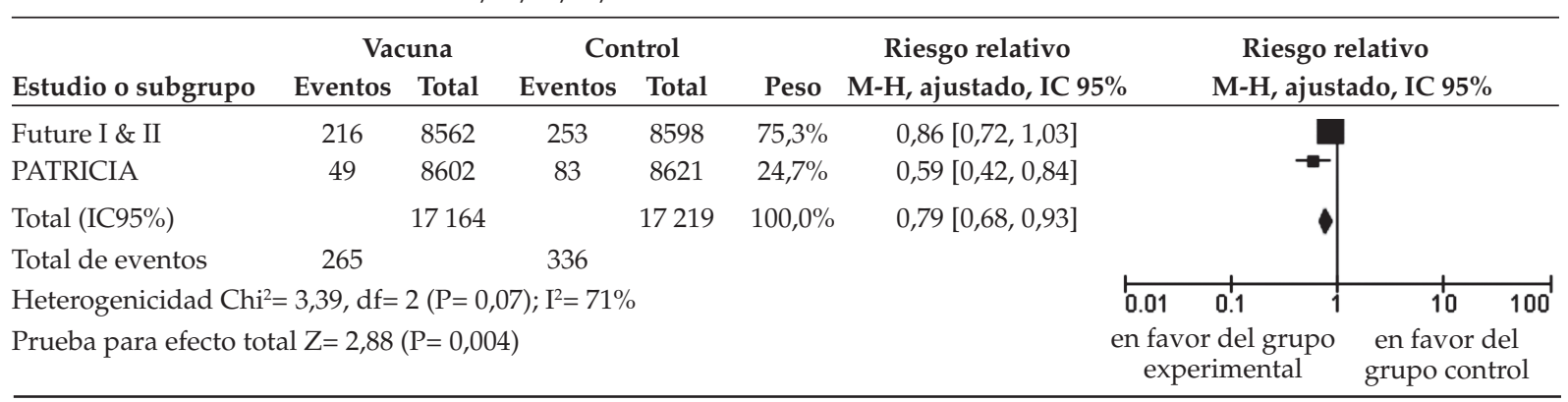


palde el reemplazo de las técnicas de pesquisa de rutina por la vacuna. Debido a que su eficacia a largo plazo para prevenir la aparición de cáncer de cuello uterino aún no ha sido completamente probada, sólo el seguimiento a largo plazo de las cohortes de pacientes vacunadas mostrarán en un futuro la eficacia real en su prevención.

Se plantea a la vacunación en la preadolescencia, en conjunto con la pesquisa mediante el PAP y la prevención de enfermedades de transmisión sexual, como la estrategia más completa y válida para la prevención del cáncer de cuello uterino. Estrategia que ya ha sido adoptada por muchos países.

La posible incorporación de la vacuna como medida de prevención primaria ha despertado gran interés en el mundo y especialmente en la región, con realización de numerosas evaluaciones económicas ${ }^{18-27}$ destinadas a evaluar la costo-eficacia de dicha intervención en diferentes países de medianos o bajos ingresos, como la Argentina, Brasil, Chile, Colombia, México y Perú. Si bien las metodologías y escenarios utilizados son dispares, en todos los estudios los resultados de costo-eficacia fueron muy dependientes del costo de la vacuna.

Los beneficios de la vacunación serían potencialmente mayores en países con baja adherencia a la pesquisa, pero dicha estrategia podría ser inasequible en países de medianos y bajos ingresos debido a los valores actuales de la vacuna.

\section{CONCLUSIÓN}

Las vacunas disponibles actualmente han demostrado ser eficaces y seguras para la prevención de lesiones CIN2+ asociadas a los serotipos 16 y 18 de HPV y conferir protección cruzada contra los serotipos $31,33,45,52$ y 58 no incluidos en la vacuna. Sin embargo, su eficacia a largo plazo para prevenir el cáncer de cuello uterino no ha sido completamente probada.

La costo-eficacia de la incorporación de la vacuna en Latinoamérica y el Caribe es muy dependiente del costo de la vacuna en cada país.

Financiación: Este metaanálisis fue elaborado como parte de un documento de evaluación de tecnologías sanitarias que se realizan gracias a los aportes de entidades públicas, organizaciones no gubernamentales, obras sociales y seguros privados de salud para el desarrollo de documentos del área de Evaluación de Tecnologías Sanitarias del Instituto de Efectividad Clínica y Sanitaria.

\section{Agradecimientos}

A todos los integrantes del área de Evaluaciones de Tecnologías Sanitarias del Instituto de
Efectividad Clínica y Sanitaria, especialmente al Bibliotecario Daniel Comandé.

\section{BIBLIOGRAFÍA}

1. WHO. GLOBOCAN. Lyon, France: World Health Organization; 2008 [Acceso: 10 de junio de 2011]. Disponible en: http://globocan.iarc.fr/.

2. zur Hausen H. Condylomata acuminata and human genital cancer. Cancer Res 1976;36(2 pt 2):794.

3. Koutsky L. Epidemiology of genital human papillomavirus infection. Am J Med 1997;102(5A):3-8.

4. Bosch FX, Manos MM, Munoz N, Sherman M, et al. Prevalence of human papillomavirus in cervical cancer: a worldwide perspective. International biological study on cervical cancer (IBSCC) Study Group. J Natl Cancer Inst 1995;87(11):796-802.

5. Ciapponi A, Bardach A, Glujovsky D, Gibbons L, Picconi MA. Type-specific HPV prevalence in cervical cancer and high-grade lesions in Latin America and the Caribbean: systematic review and meta-analysis. PLoS ONE 2011;6(10):e25493.

6. UNICEF.Immunization Summary, Astatistical referencecontaining data through 2009. New York, USA: World Health Organization; 2011. [Acceso: 7 de julio de 2012]. Disponible en: http: / / www.childinfo.org/files/32775_UNICEF.pdf.

7. Sawaya GF, Smith-McCune K. HPV vaccination--more answers, more questions. N Engl J Med 2007;356(19):1991-3.

8. Miller NB, Raychaudhuri G, Toerner JG. Human papillomavirus vaccine. N Engl J Med 2007;357(11):1154-5; author reply 5-6.

9. ASCUS-LSIL, Traige, Study, (ALTS), Group. Results of a randomized trial on the management of cytology interpretations of atypical squamous cells of undetermined significance. Am J Obstet Gynecol 2003;188(6):1383-92.

10. Pagliusi SR, Teresa Aguado M. Efficacy and other milestones for human papillomavirus vaccine introduction. Vaccine 2004;23(5):569-78.

11. Wright TC, Jr., Cox JT, Massad LS, Carlson J, et al. 2001 consensus guidelines for the management of women with cervical intraepithelial neoplasia. Am J Obstet Gynecol 2003;189(1):295-304.

12. Paavonen J, Naud P,Salmeron J, Wheeler CM, et al. Efficacy of human papillomavirus (HPV)-16/18 AS04-adjuvanted vaccine against cervical infection and precancer caused by oncogenic HPV types (PATRICIA): final analysis of a double-blind, randomised study in young women. Lancet 2009;374(9686):301-14.

13. Wheeler CM, Kjaer SK, Sigurdsson K, Iversen OE, et al. The impact of quadrivalent human papillomavirus (HPV; types $6,11,16$, and 18) L1 virus-like particle vaccine on infection and disease due to oncogenic nonvaccine HPV types in sexually active women aged 16-26 years. J Infect Dis 2009;199(7):936-44.

14. Harper DM, Franco EL, Wheeler CM, Moscicki AB, et al. Sustained efficacy up to 4.5 years of a bivalent L1 viruslike particle vaccine against human papillomavirus types 16 and 18: follow-up from a randomised control trial. Lancet 2006;367(9518):1247-55.

15. Muñoz N, Kjaer SK, Sigurdsson K, Iversen OE, et al. Impact of human papillomavirus (HPV)-6/11/16/18 vaccine on all HPV-associated genital diseases in young women. $J$ Natl Cancer Inst 2010;102(5):325-39.

16. Garland SM, Hernández-Avila M, Wheeler CM, Pérez G, et al. Quadrivalent vaccine against human papillomavirus to prevent anogenital diseases. N Engl J Med 2007; 356(19):1928-43. 
17. Future, II, Study, Group. Quadrivalent vaccine against human papillomavirus to prevent high-grade cervical lesions. N Engl J Med 2007;356(19):1915-27.

18. GoldieSJ, O'Shea M, DíazM, KimSY. Benefits, cost requirements and cost-effectiveness of the HPV16,18 vaccine for cervical cancer prevention in developing countries: policy implications. Reproduct Health Matters 2008;16(32):86-96.

19. Goldie SJ, Kim JJ, Kobus K, Goldhaber-Fiebert JD, et al. Cost-effectiveness of HPV 16, 18 vaccination in Brazil. Vaccine 2007;25(33):6257-70

20. Goldie SJ, Díaz M, Constenla D, Alvis N, et al. Mathematical Models of Cervical Cancer Prevention in Latin America and the Caribbean. Vaccine 2008;26(Suppl. 11):L59-L72.

21. Colantonio L, Gómez JA, Demarteau N, Standaert B, et al. Cost-effectiveness analysis of a cervical cancer vaccine in five Latin American countries. Vaccine 2009;27(40):5519-29.

22. Gutiérrez-Delgado C, Báez-Mendoza C, González-Pier E, de la Rosa AP, Witlen R. Relación costo-efectividad de las intervenciones preventivas contra el cáncer cervical en mujeres mexicanas: resultados de un modelo markoviano desde la perspectiva del sector público. Sal Publ Mex 2008;50(2):107-18.

23. Insinga RP, Dasbach EJ, Elbasha EH, Puig A, Reynales-Shigematsu LM. Cost-effectiveness of quadrivalenthuman papillomavirus (HPV) vaccination in Mexico: A transmission dynamic model-based evaluation. Vaccine 2007;26(1):128-39.

24. Reynales-Shigematsu LM, Rodrígues ER, Lazcano-Ponce E. Cost-effectiveness analysis of a quadrivalent human papilloma virus vaccine in Mexico. Arch Med Res 2009; 40(6):503-13.

25. Kim JJ, Andres-Beck B, Goldie SJ. The value of including boys in an HPV vaccination programme: A cost-effectiveness analysis in a low-resource setting. $\mathrm{Br}$ J Cancer 2007;97(9):1322-8.

26. De La Hoz F, Alvis N, Narvez J, Gamboa O, Chocont A. Cost-effectiveness of human papillomavirus vaccine in Colombia in 2007. Value Health 2009;12 (3):A42.

27. Demarteau N, Suárez E, Gómez J, Standaert B. Modelling the impact of different vaccine profiles on the cost-effectiveness of HPV vaccination in the Chilean setting. Int J Gyn Obst 2009;107:S673-S4.

Sólo quienes sean capaces de encarnar la utopía, serán aptos para el combate decisivo, el de recuperar cuanto de humanidad hayamos perdido.

ERNESTO SÁBATO 